\title{
Coil-free active stabilisation of extended payloads with optical inertial sensors
}

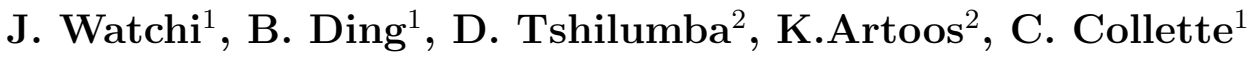 \\ ${ }^{1}$ Universite Libre de Bruxelles, BEAMS department, 50, F.D. avenue, 1050 Brussels \\ (Belgium) \\ ${ }^{2}$ European Organization for Nuclear Research (CERN), Engineering department, \\ Geneva 23, 1211 Geneva (Switzerland) \\ E-mail: jennifer.watchi@ulb.ac.be
}

\begin{abstract}
This paper presents a new active isolation strategy and system which is dedicated to extended payloads, and compatible with particle accelerator environment. In comparison to the current isolation systems used in this environment, the system proposed does not contains any coil or elastomer and the supporting frame is dedicated to isolate long payloads from seismic motion. The concept proposed has been tested numerically on 3 and 6 degrees of freedom models, and validated experimentally on a one degree of freedom scaled test set-up. An attenuation of $40 \mathrm{~dB}$ at $1 \mathrm{~Hz}$ has been reached with the stage built. The complete description of performance and a noise budgeting are included in this paper.
\end{abstract}

\section{Introduction}

Effective isolation from ground vibration is required for many applications including lithography machines [1], atomic force microscopy [2], atomic gravimetry [3], medical imaging, and large instruments dedicated to experimental physics [4]. In fact, ground motion includes microseismic waves that have an amplitude of the order of $10^{-6} \mathrm{~m}$. Consequently, if ground motion is transmitted to a precision tool, it constitutes one of the most limiting factor to the resolution of the system [5]. Ground motion attenuation is particulary challenging for the stabilization of the electromagnets used to guide particle colliders beams. In fact, if the two beams are not perfectly aligned, the collision rate, called luminosity, will drastically decrease. Beyond this stringent requirement, several additional constraints must be adequately addressed. They are related to the extended shape of the structure, the limited stability of the floor on which they are mounted, and limitations on the technology of the sensors and actuators which could couple with the electromagnets. During the last twenty years, many studies have been conducted in order to comply with these requirements, both for the active stabilisation of the main beam quadrupoles [6-8], and for the stabilisation of final focus quadrupoles [9-15]. A critical review of strategies can be found in [16]. Over 
the years, the level of stability has been continuously improved, reaching the required values and even better. However so far, to the best of the authors' knowledge, all of them are using electromagnetic sensors for inertial control at low frequency, which are not compatible with the environment of particle colliders [17]. Other sensitive seismic sensors exist but they also measure some parasitic signal due to the surrounding magnetic field and the particle beam; either the sensor flexure is build in a ferromagnetic material [5] or it includes elastomer or eletronics [18] which are damaged by the highenergy particle beam. New sensors based on an interferometric readout have been developed which can provide an accurate signal in this harsh environment [19-21].

In this paper, the control approach proposed is the use of a high resolution optical inertial sensor and a mechatronic architecture dedicated to support long and extended objects like electromagnets.

While this control strategy is specifically designed for particle accelerators, this isolation system is needed for other precision measurement tools that work in a similar environment; for example, atomic gravimeters use electromagnetic fields to generate the Raman transitions [3] and medical imaging either use strong magnetic fields or particle beams to scan a body. In addition, several of the features and components of the instrumentation are also of potential interest for the LIGO in-vacuum isolators. Their performance is currently partly limited by the sensor noise of its vertical inertial sensors at low frequency and by the control bandwidth at high frequency $[22,23]$.

The paper is organised as follows. Section two presents the supporting concept proposed, along with a justification for this architecture. Section three discusses in detail the performance on a simplified three degrees of freedom (d.o.f.) model. Section four extends the study to a six d.o.f. model, and section five contains an experimental validation. The conclusions are given in section six.

\section{Concept}

In order to control the six degrees of freedom of a sensitive equipment, at least six actuators are required. Depending on the feedback control objective, the supports are oriented parallel or perpendicular to the gravity [24,25], or inclined in the manner of a Stewart platform [26]. For the quadrupoles of a particle accelerator, a high control authority is required in the vertical direction and a weaker authority in the lateral, yaw and pitch directions. This is due to the cross section of the beam. No authority is required in roll and longitudinal directions. Due to the extended shape of the electromagnet, it has been decided to use eight supports, as shown in green in Fig. 1: four in the vertical direction (two at each end), and four in the lateral direction, with a small angle to restrict the longitudinal motion.

At each end of the structure, vertical and lateral inertial sensors (indicated by box marked S) are used to drive the actuators. Each sensor drives two actuators. Each end is controlled independently. For this reason, the concept is studied in two steps, 
presented in the two following sections. Section three presents the control strategy used for only one end applied to a three degrees of freedom system (vertical, lateral, roll). The controller will be presented, along with an analysis of the sensitivity to some geometrical parameters. Section four extends the study to the full (6 d.o.f.) system.

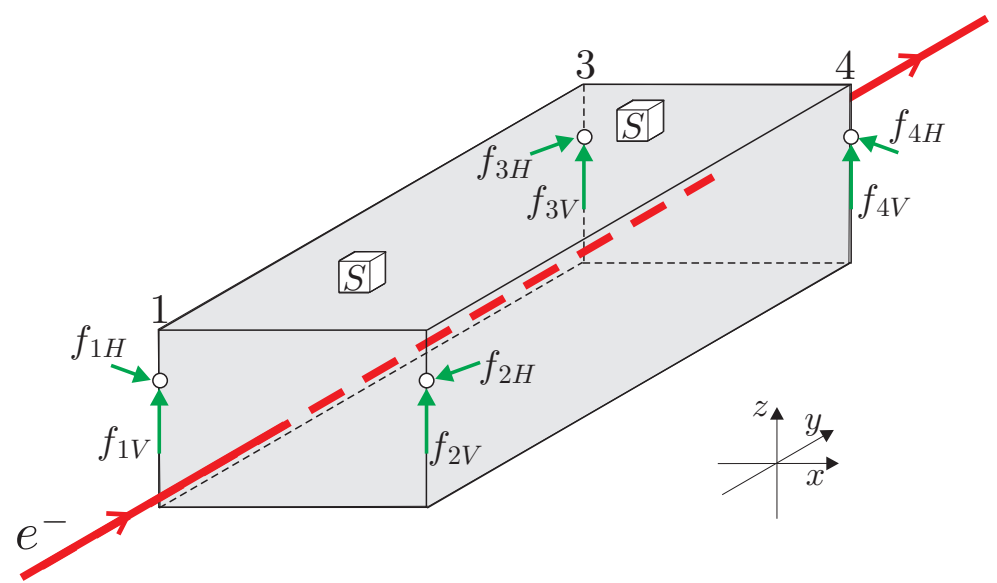

$(a)$

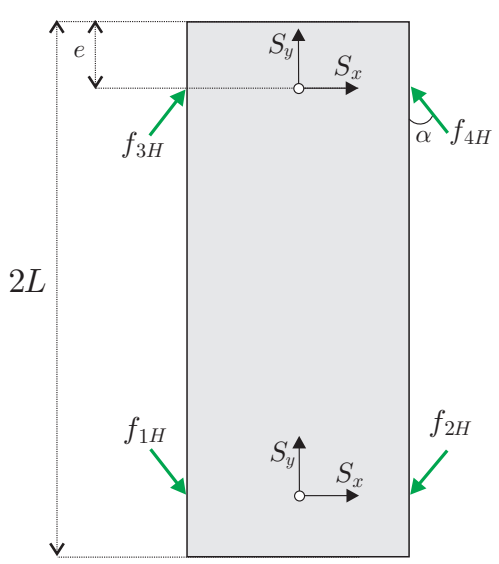

(b)

Figure 1. (a) General view of the quadrupole with the eight legs used for the control. With each set of four legs, a sensor is positioned. It measures the motion in the $x, y$ and $z$ directions. The electron beam is in red and goes in the $y$ direction. (b) Top view of the quadrupole. The four horizontal legs are tilted with an angle $\alpha$.

\section{Three d.o.f. model}

\subsection{Mechanical design}

Figure 2 is the xz cross section of the extended structure in the control system plane. Four actuators are used, two in the vertical direction and two in the horizontal direction. Moreover, neither the sensor nor the actuators are located at the center of mass. The parameter $l$ corresponds to the distance between the center of mass and the sensor in the $z$ direction. The sensor is assumed to be centred in the $x$ direction. The parameters $d$ and $h$ represent respectively the distance between the center of mass and the actuator in the $x$ and $z$ direction. These misalignments are of importance for the stability of the control system. The influence of these parameters on the stabilization of the electromagnet is the objective of this first study. Taking into account the actuators and the ground motion, the equation of motion of the system is

$$
M \ddot{X}+C \dot{X}+K X=k_{a} B w+B f_{a}
$$

$$
\text { where } M=\left(\begin{array}{ccc}
m & 0 & 0 \\
0 & m & 0 \\
0 & 0 & I_{z_{G}}
\end{array}\right), C=c_{a}\left(\begin{array}{ccc}
2 & 0 & -2 h \\
0 & 2 & 0 \\
-2 h & 0 & 2 d^{2}+2 h^{2}
\end{array}\right) \text {, }
$$




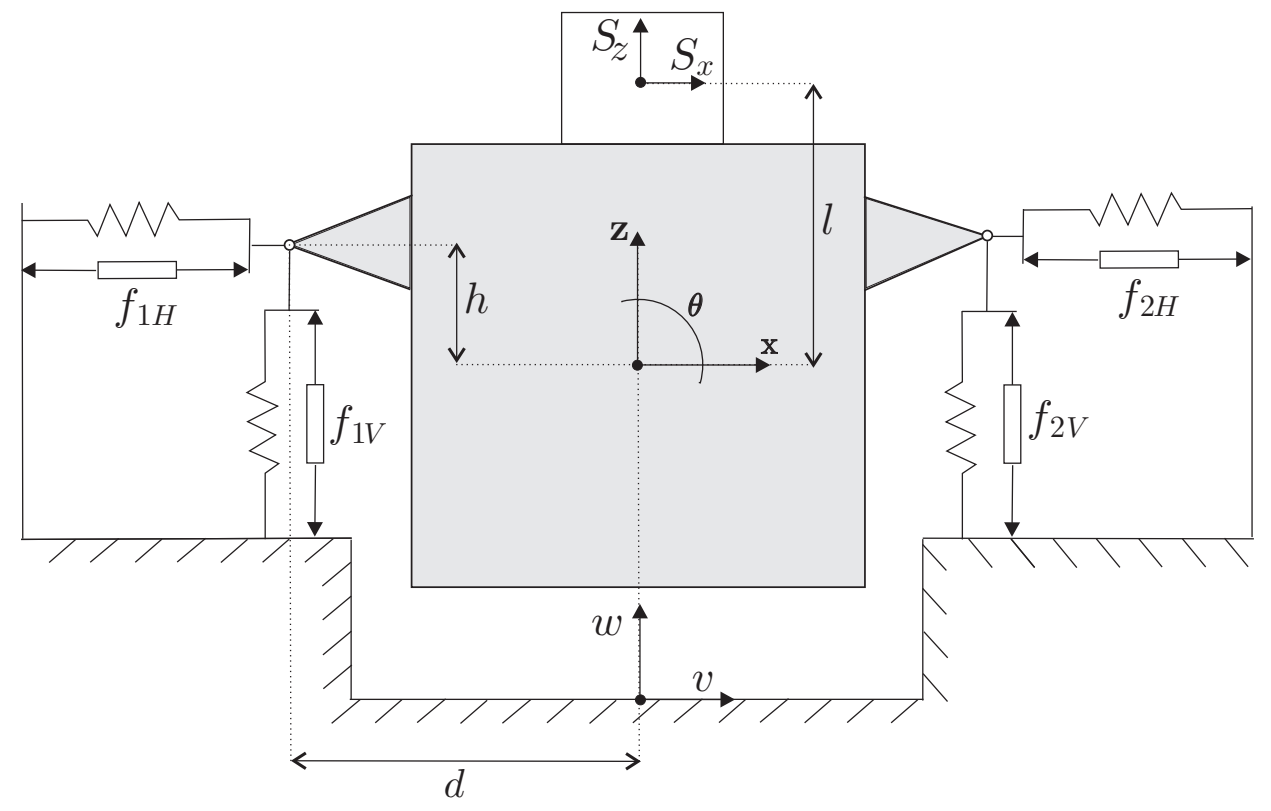

Figure 2. Representation of the 3 d.o.f. experiment. Each actuator is in parallel to a spring with a stiffness $k_{a}$. The sensor used measures the $x$ and $z$ motions. The distance between the center of mass and the sensor, $l$, the actuator in the $x$ direction, $d$, and the actuator in the $z$ direction, $h$, are shown.

$K=k_{a}\left(\begin{array}{ccc}2 & 0 & -2 h \\ 0 & 2 & 0 \\ -2 h & 0 & 2 d^{2}+2 h^{2}\end{array}\right), B=J^{T}, J=\left(\begin{array}{ccc}0 & 1 & -d \\ 0 & 1 & d \\ 1 & 0 & -h \\ -1 & 0 & h\end{array}\right)$

and $X^{T}=\left(\begin{array}{lll}x & z & \theta\end{array}\right), w^{T}=\left(\begin{array}{ll}v & w\end{array}\right)$ and $f_{a}^{T}=\left(\begin{array}{llll}f_{1 V} & f_{2 V} & f_{1 H} & f_{2 H}\end{array}\right)$.

$M$ is the mass matrix, $m$ is the mass of the equipment and $I_{z_{G}}$ is the inertia calculated at the mass center. $C$ and $K$ are the damping and stiffness matrices $\left(c_{a}\right.$ and $k_{a}$ are the damping and stiffness in each leg). For simplicity, we have assumed that the damping matrix is proportional to the stiffness matrix. $J$ is the Jacobian matrix, projecting the directions of the supports into the frame of the payload $X, B$ is the transposed of the Jacobian matrix $J ; w$ is the vector of ground motion in the direction of the supports. One can note that only two parameters have an influence on the system dynamics: $d$ and $h$. The Laplace Transform of equation (1) gives

$$
\begin{gathered}
\left(M s^{2}+C s+K\right) X=k_{a} B w+B f_{a} \\
\left(\begin{array}{ccc}
m s^{2}+2\left(k_{a}+c_{a} s\right) & 0 & -2 h\left(k_{a}+c_{a} s\right) \\
0 & m s^{2}+2\left(k_{a}+c_{a} s\right) & 0 \\
-2 h\left(k_{a}+c_{a} s\right) & 0 & I_{z_{G}} s^{2}+\left(2 d^{2}+2 h^{2}\right)\left(k_{a}+c_{a} s\right)
\end{array}\right) X \\
=\left(\begin{array}{cccc}
0 & 0 & 1 & -1 \\
1 & 1 & 0 & 0 \\
-d & d & -h & h
\end{array}\right)\left(\begin{array}{c}
k_{a} w+f_{1 V} \\
k_{a} w+f_{2 V} \\
k_{a} v+f_{1 H} \\
k_{a} v+f_{2 H}
\end{array}\right)
\end{gathered}
$$


where the poles of the open loop transfer function are given by

$$
\left(m s^{2}+2\left(k_{a}+c_{a} s\right)\right)\left|\begin{array}{cc}
m s^{2}+2\left(k_{a}+c_{a} s\right) & -2 h\left(k_{a}+c_{a} s\right) \\
-2 h\left(k_{a}+c_{a} s\right) & I_{z_{G}} s^{2}+\left(2 d^{2}+2 h^{2}\right)\left(k_{a}+c_{a} s\right)
\end{array}\right|=0
$$

The system has three complex poles: one corresponding to the resonance in the vertical direction, and two complex conjugate poles that depend on the system configuration, coupling the horizontal $(\mathrm{x})$ and angular $(\theta)$ motion. Figure 3 and 4 shows the evolution of these poles when the distance $h$ (figure 3) and $d$ (figure 4 ) vary. The third pole is not shown as it is always cancelled out by a zero. The coupling disappears when $h=0$. In that case, the eigen frequencies are $\Omega_{1}=\Omega_{2}=\sqrt{2 k_{a} / m}$ for the horizontal $(\mathrm{x})$ and vertical (z) modes, $\Omega_{3}=\sqrt{2 d^{2} k_{a} / I_{z_{G}}}=d \sqrt{2 k_{a} / I_{z_{G}}}$ for the rotational mode $(\theta)$. By comparison between the two figures, the parameter $d$ has less impact than the parameter $h$.

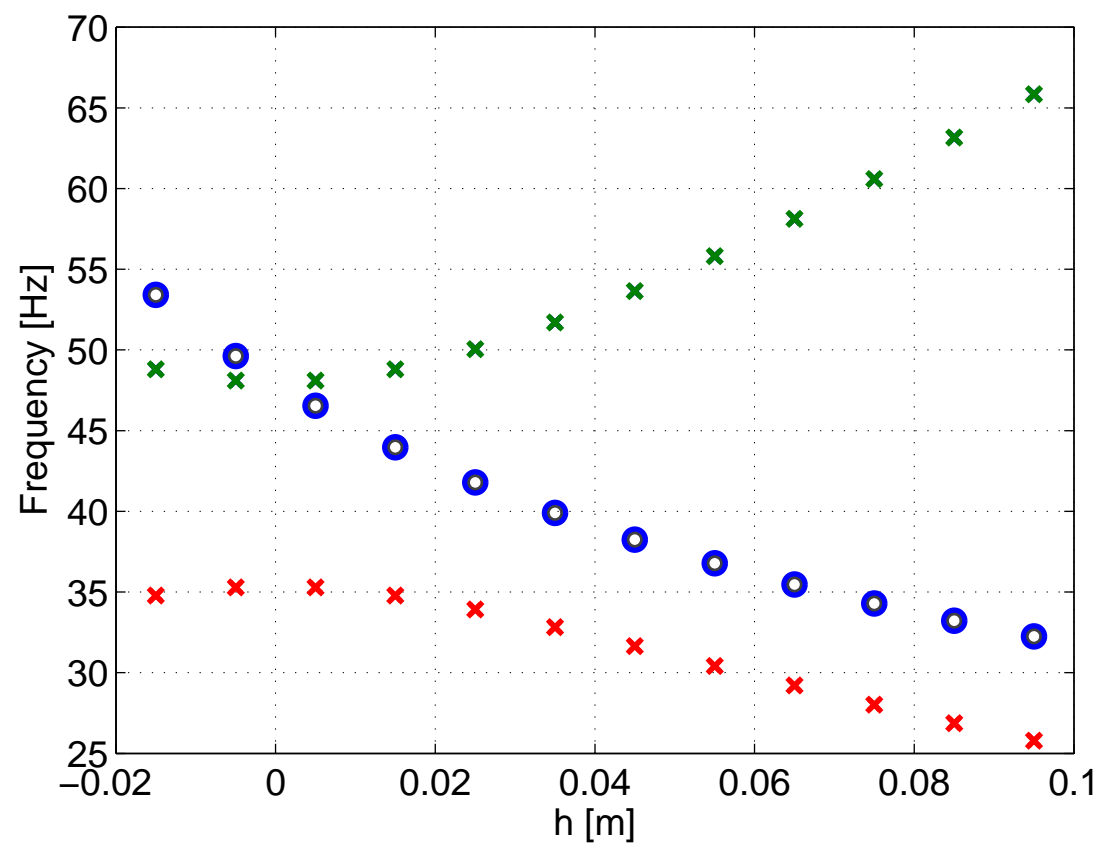

Figure 3. Frequency evolution of the poles and zeros depending on $h$, the distance between the center of mass and the actuator position in the $z$ direction. The poles are marked with a cross ' $\mathrm{x}$ ' and the zeros with a circle 'o'. The parameter $d$ has been set to $0.12 \mathrm{~m}$.

In order to have alternating poles and zeros which guarantees a stable loop, the parameter $h$ needs to be positive. In addition, as there is no benefit to position the legs at a distance larger than the structure dimensions, $h$ is set small. Consequently, in the next section, the parameter $h$ will be set to $0.05 \mathrm{~m}$ which corresponds to the position of the legs on the experimental structure. The distance $d$ will be $0.12 \mathrm{~m}$ which is half the length of the structure in the $x$ direction. 


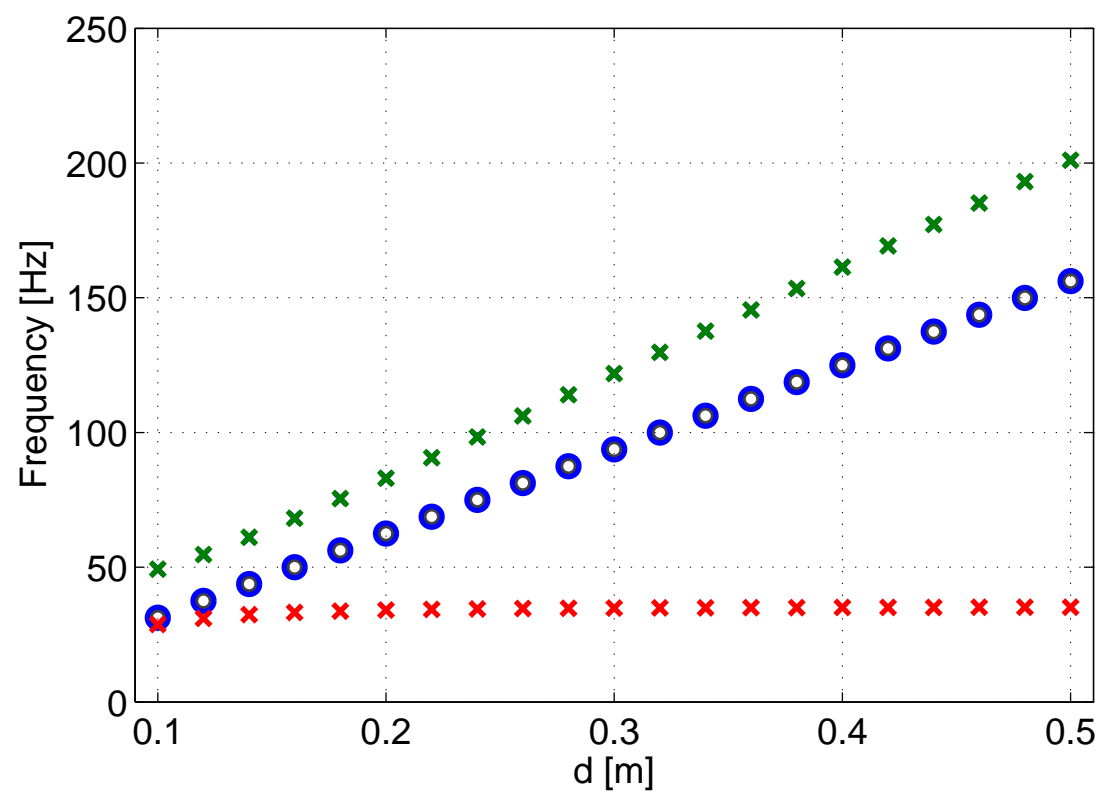

Figure 4. Frequency evolution of the poles and zeros depending on $d$, the distance between the center of mass and the actuator position in the $x$ direction. The poles are marked with a cross ' $\mathrm{x}$ ' and the zeros with a circle 'o'. The parameter $h$ has been set to $0.05 \mathrm{~m}$.

\subsection{Inertial control}

The electromagnet has to be stabilized in the vertical and the lateral directions. In fact, the roll around the longitudinal axis is not responsible of beam deviation. Therefore, inertial control will be applied only in the $x$ and $z$ direction, as shown in figure 5 . However, coupling between the horizontal and tilt motion has an influence on the control performance: the control force applied to one of these two directions will have an impact on the other one. This section highlights theoretically this coupling.

Assuming that the vector $f_{a}$ contains the forces exerted by the four actuators, as defined in section 3.1, we have

$$
F^{T}=\left(B f_{a}\right)^{T}=\left(\begin{array}{lll}
f_{x} & f_{z} & f_{\theta}
\end{array}\right)
$$

where $F$ is the vector of forces applied by the actuators along the three d.o.f. of the system. As the width of the equipment is small (i.e. the separation between the two vertical supports) and the width of an inertial sensor is large, a single vertical sensor is used to control the four actuators. Calling $S=\left(\begin{array}{c}S_{x} \\ S_{z}\end{array}\right)$ the vector containing the signals of the inertial sensor in the vertical and lateral directions, the vector of control forces is expressed as

$$
f_{a}=H_{c} S
$$


where $H_{c}=-g k_{a}\left(\begin{array}{cc}0 & H_{z} \\ 0 & H_{z} \\ H_{x} & 0 \\ -H_{x} & 0\end{array}\right)$, g is the controller gain, $H_{x}$ is the controller in the $x$ direction and $H_{z}$ in the $z$ direction. Neglecting the dynamics of the sensors for simplicity, the sensors signals can be converted back in equipment displacement with

$$
S=\left(\begin{array}{c}
S_{x} \\
S_{z}
\end{array}\right)=T X=\left(\begin{array}{ccc}
1 & 0 & -l \\
0 & 1 & 0
\end{array}\right)\left(\begin{array}{l}
x \\
z \\
\theta
\end{array}\right)
$$

Combining the definition of $f_{a}, H_{c}$ and $S$, we have

$$
\begin{gathered}
f_{a}=H_{c} T X=-g k_{a}\left(\begin{array}{cc}
0 & H_{z} \\
0 & H_{z} \\
H_{x} & 0 \\
-H_{x} & 0
\end{array}\right)\left(\begin{array}{ccc}
1 & 0 & -l \\
0 & 1 & 0
\end{array}\right)\left(\begin{array}{c}
x \\
z \\
\theta
\end{array}\right)=-g k_{a}\left(\begin{array}{ccc}
0 & H_{z} & 0 \\
0 & H_{z} & 0 \\
H_{x} & 0 & -l H_{x} \\
-H_{x} & 0 & l H_{x}
\end{array}\right)\left(\begin{array}{l}
x \\
z \\
\theta
\end{array}\right) \\
F=-B g H k_{a} T X=-g k_{a} B H T X=-g k_{a} H\left(\begin{array}{ccc}
2 & 0 & -2 l H_{x} \\
0 & 2 H_{z} & 0 \\
-2 h H_{x} & 0 & 2 h l H_{x}
\end{array}\right)\left(\begin{array}{c}
x \\
z \\
\theta
\end{array}\right)
\end{gathered}
$$

The above relation clearly shows the coupling between the horizontal and tilt d.o.f. as predicted.

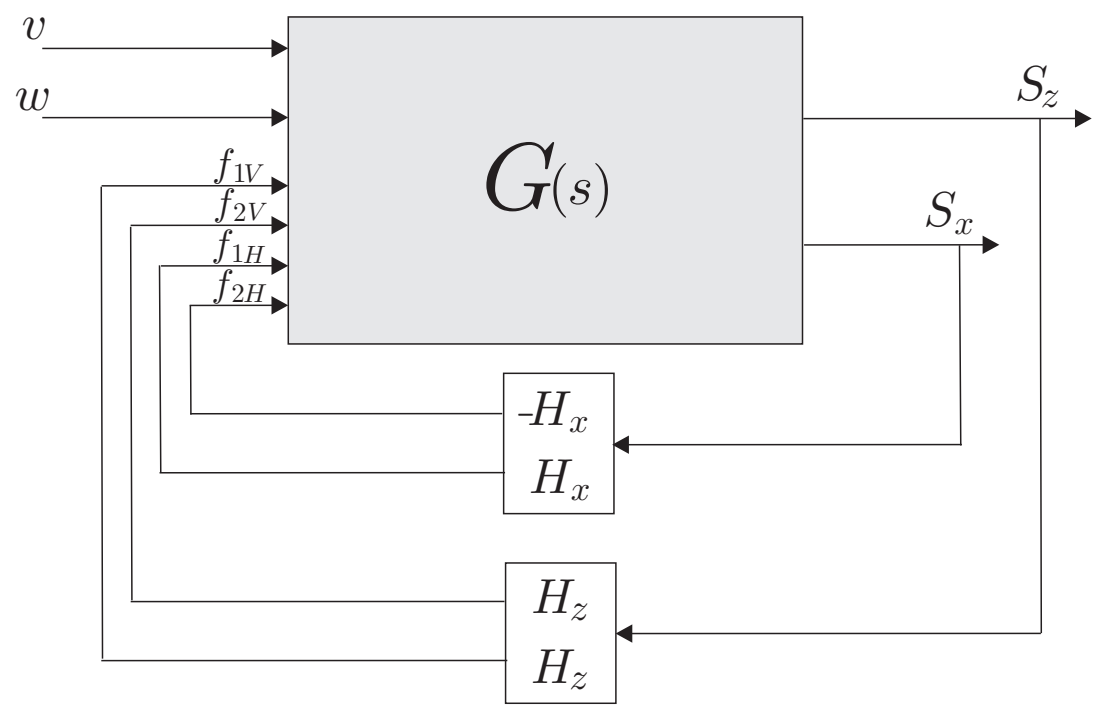

Figure 5. Block diagram of the control loop for the 3 d.o.f. model: the inputs are the ground motion and the actuator forces and the outputs are the two signals from the sensor. A vertical and horizontal controller can be set independently.

\subsection{Controller design}

Based on the transfer function between horizontal and vertical pairs of sensor/actuator, a single stable controller has been designed (Fig. 6). The controller is a combination of 
two lags and one lead:

$$
H=\frac{s+z_{1}}{s+p_{1}} \frac{s+z_{2}}{s+p_{2}} \frac{s+z_{3}}{s+p_{3}}
$$

with $p_{1}=2 \pi 0.2632 \mathrm{rad} / \mathrm{s}, z_{1}=2 \pi 10.93 \mathrm{rad} / \mathrm{s}, p_{2}=2 \pi 5 \mathrm{rad} / \mathrm{s}, z_{2}=2 \pi 20 \mathrm{rad} / \mathrm{s}, p_{3}$ $=2 \pi 60 \mathrm{rad} / \mathrm{s}$ and $z_{3}=2 \pi 10 \mathrm{rad} / \mathrm{s}$.

The stability margins are shown in figure 6.(a) and (b) respectively for the horizontal and vertical motion. Phase margins around $15^{\circ}$ have been reached for both directions. Obviously, larger margins can be obtained by moving the lead to larger frequencies.

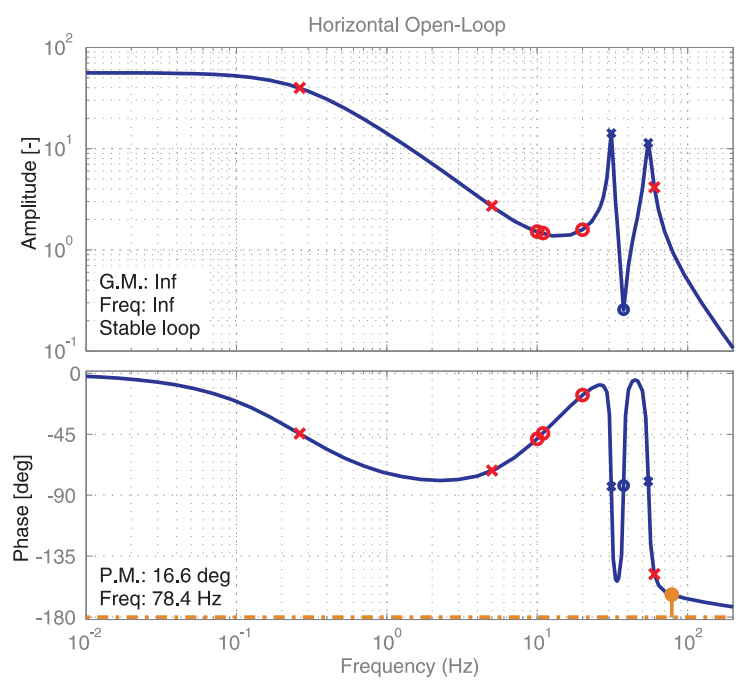

(a)

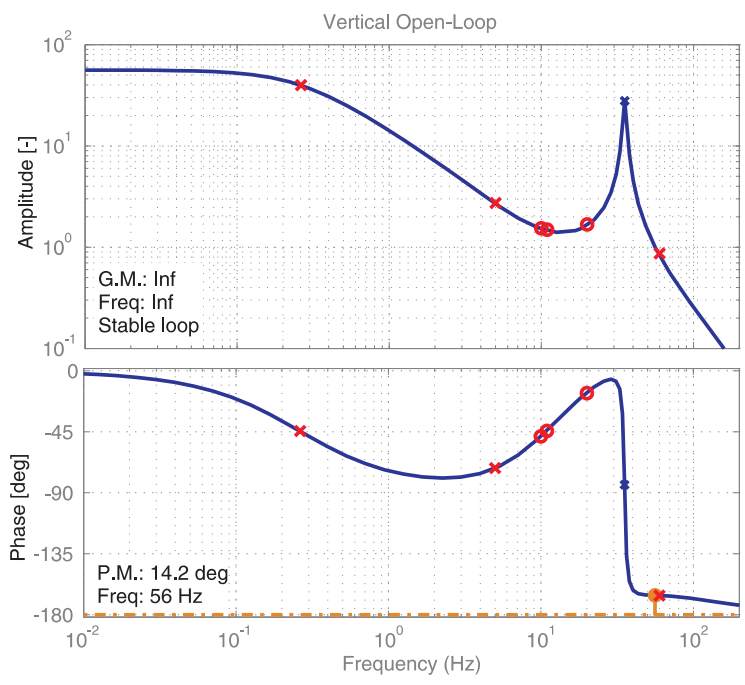

(b)

Figure 6. Bode diagram representation of the open loop transfer functions of the 3 d.o.f. model for the horizontal (a) and vertical (b) motions. Both feedback loop are stable.

The transmissibility between ground and structure motion is shown in figure 7.(a) and (b). For the simulation, the following numerical values have been used: $m=10 \mathrm{~kg}$, $k_{a}=2.5 \times 10^{5} \mathrm{~N} / \mathrm{m}, c_{a}=50 \mathrm{Ns} / \mathrm{m}, h=0.05 \mathrm{~m}, d=0.12 \mathrm{~m}$ and the gain applied is $10^{6}$. The motion of the structure has been damped by a factor 30 at $1 \mathrm{~Hz}$ and by a factor 100 at $0.1 \mathrm{~Hz}$ in both directions.

\section{Six d.o.f. model}

\subsection{Mechanical design}

In order to consider the complete extended structure in figure 1, a 6 d.o.f. system has been developed. Four actuators are positioned at each end of the structure. To control them, two sensors are also placed, one at each end. The matrices used in the equation of motion, eq. (1), become 


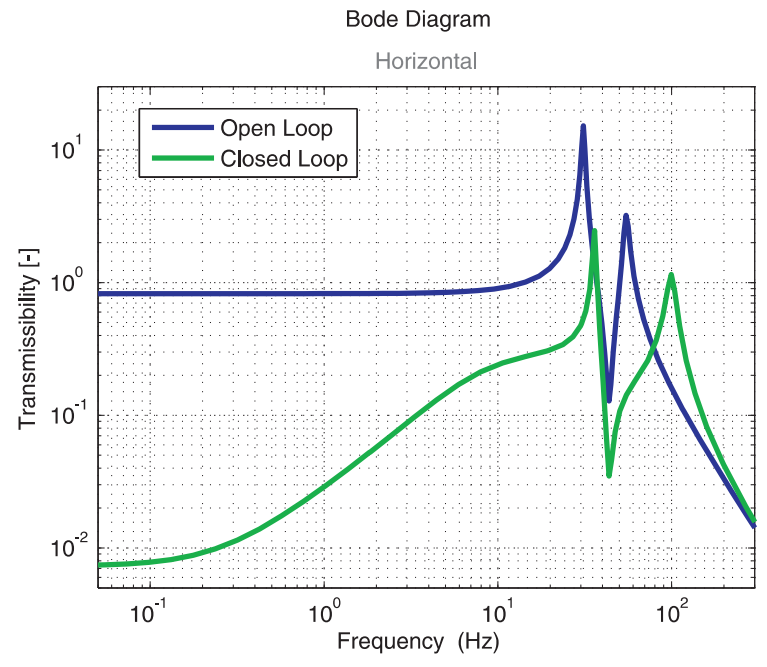

(a)

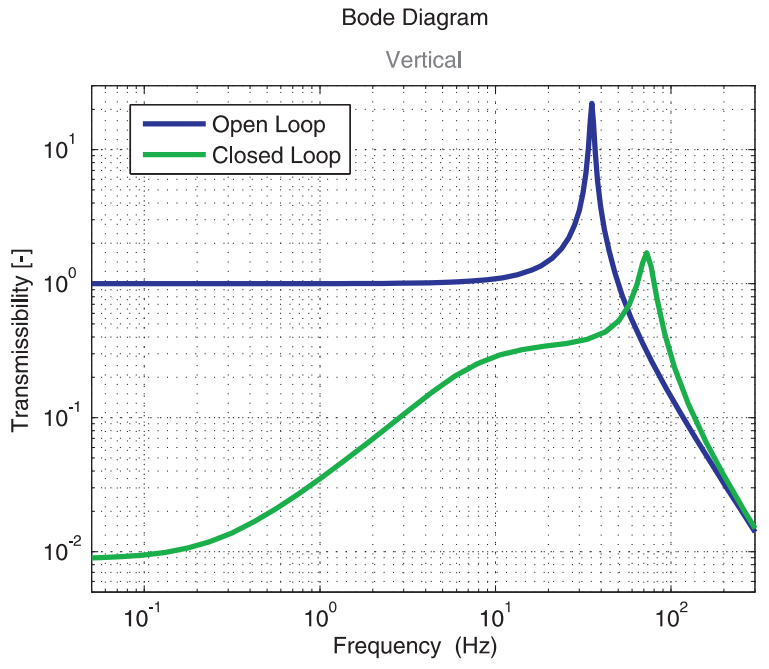

(b)

Figure 7. Transmissibility between the horizontal (a) and vertical (b) structure motion and its corresponding ground motion for the 3 d.o.f. model. The blue curve corresponds to the controlled system and the green one to the closed loop.

$$
\begin{aligned}
M & =\left(\begin{array}{cccccc}
m & 0 & 0 & 0 & 0 & 0 \\
0 & m & 0 & 0 & 0 & 0 \\
0 & 0 & m & 0 & 0 & 0 \\
0 & 0 & 0 & I_{x} & 0 & 0 \\
0 & 0 & 0 & 0 & I_{y} & 0 \\
0 & 0 & 0 & 0 & 0 & I_{z}
\end{array}\right), K=k_{a} B J, C=c_{a} B J \\
J & =\left(\begin{array}{cccccc}
\sin \alpha & -\cos \alpha & 0 & h \cos \alpha & h \sin \alpha & d \cos \alpha+(L-e) \sin \alpha \\
-\sin \alpha & -\cos \alpha & 0 & h \cos \alpha & -h \sin \alpha & -d \cos \alpha-(L-e) \sin \alpha \\
\sin \alpha & \cos \alpha & 0 & -h \cos \alpha & h \sin \alpha & -d \cos \alpha-(L-e) \sin \alpha \\
-\sin \alpha & \cos \alpha & 0 & -h \cos \alpha & -h \sin \alpha & d \cos \alpha+(L-e) \sin \alpha \\
0 & 0 & 1 & -L & d & 0 \\
0 & 0 & 1 & -L & -d & 0 \\
0 & 0 & 1 & L & d & 0 \\
0 & 0 & 1 & L & -d & 0
\end{array}\right)
\end{aligned}
$$

and $X^{T}=\left(\begin{array}{llllll}x & y & z & \theta_{x} & \theta_{y} & \theta_{z}\end{array}\right)$,

$w^{T}=\left(\begin{array}{llllllll}w_{1 H} & w_{2 H} & w_{3 H} & w_{4 H} & w_{1 V} & w_{2 V} & w_{3 V} & w_{4 V}\end{array}\right)$,

$f_{a}^{T}=\left(\begin{array}{llllllll}f_{1 H} & f_{2 H} & f_{3 H} & f_{4 H} & f_{1 V} & f_{2 V} & f_{3 V} & f_{4 V}\end{array}\right)$

$L$ is the half length of the extended structure in the $y$ direction, $e$ is the distance between the extremity of the structure and the actuator position in the $y$ direction. As the horizontal actuators are not perpendicular to the structure, the projection on the two horizontal axes is taken into account thanks to the angle $\alpha$, as shown in figure 1.(b). 
The ground motion is projected in the direction of the horizontal and vertical legs $\left(w_{i H}\right.$ and $\left.w_{i V}, i=1,2,3,4\right)$. The actuation force vector $f_{a}$ is defined in the same way.

The distance between the center of mass and the actuator legs $d$ and $h$ remain the same as in section 3 .

\subsection{Inertial control}

Again, only the $x$ and $z$ directions will be controlled as motion along the longitudinal axis does not induce any beam deviation. Two inertial sensors are used, they are located at both extremity of the structure. The vector containing the signal of one inertial sensor is $S^{T}=\left(\begin{array}{lll}S_{x} & S_{y} & S_{z}\end{array}\right)$. One sensor is used to control one extremity of the structure similarly to what is explained in section 3.2 .

\subsection{Controller design}

The same controller as the one described in eq. (10) is used for the vertical and horizontal stabilization. At each end, the isolation is performed thanks to the sensor and the four actuators. The controller leads to slightly smaller phase margins, as seen in figure 8 . As the whole structure is now considered, the parameters used for the simulation are slightly different. The numerical values used are now: $m=20 \mathrm{~kg}, k_{a}=5 \times 10^{5} \mathrm{~N} / \mathrm{m}$, $c_{a}=100 \mathrm{Ns} / \mathrm{m}, h=0.05 \mathrm{~m}, d=0.12 \mathrm{~m}$ and the gain applied is $2 \times 10^{6}$. In addition, the angle $\alpha$ of the horizontal legs is set to $20^{\circ}$, see figure 1.(b). Figure 9 shows that the controller now attenuates the motion at $1 \mathrm{~Hz}$ by a factor of 30 in the vertical direction and by a factor of 80 in the horizontal direction.

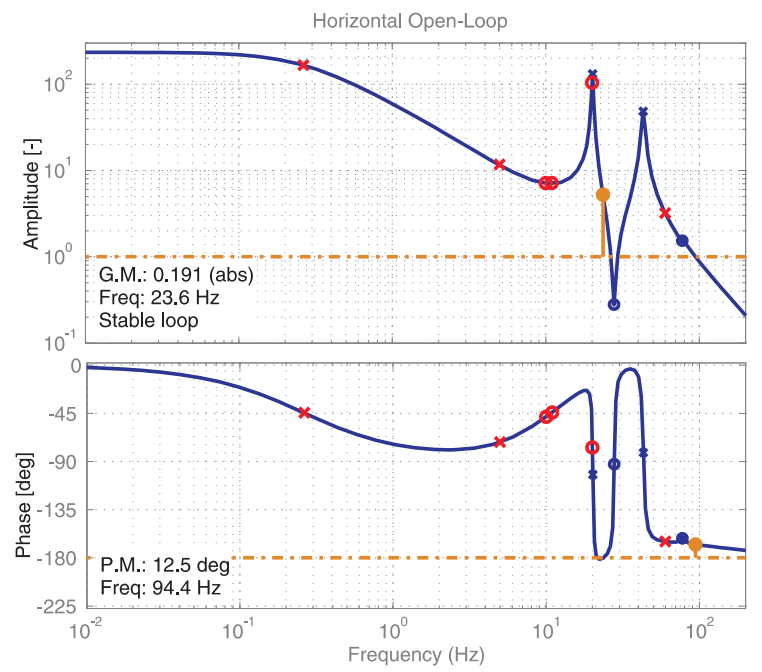

(a)

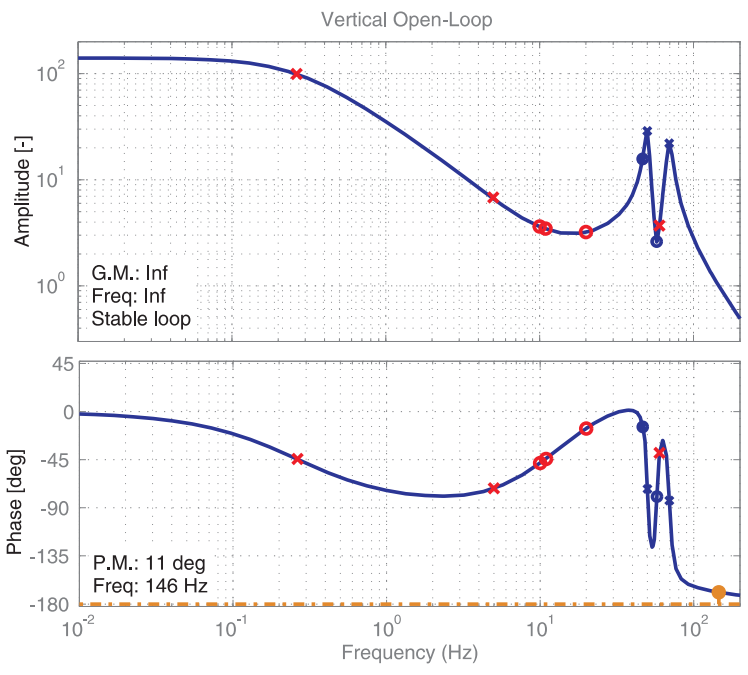

(b)

Figure 8. Bode diagram representation of the open loop transfer functions of the 6 d.o.f. model for the horizontal (a) and vertical (b) motion. Both feedback loops are stable. 




(a)

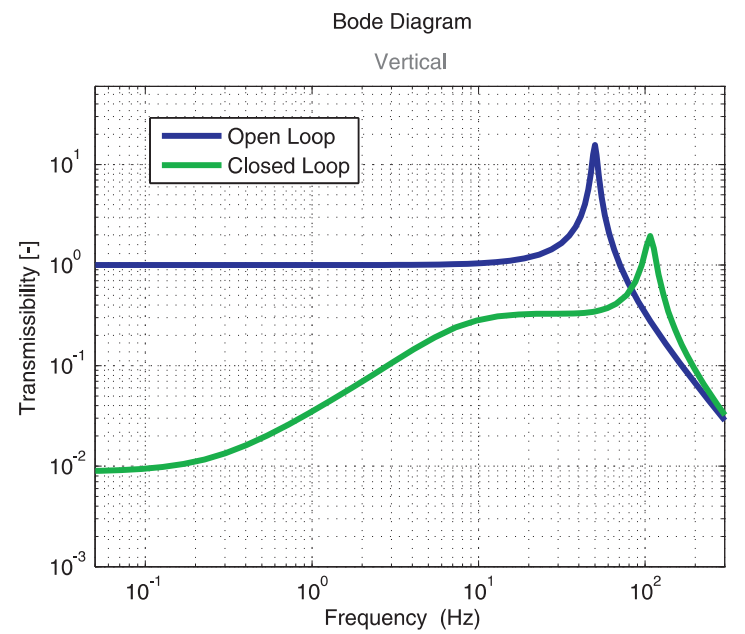

(b)

Figure 9. Transmissibility of the 6 d.o.f. model: transfer function between the horizontal (a) and vertical (b) structure motion and its corresponding ground motion. The blue curve corresponds to the controlled system and the green one to the closed loop.

\section{Experimental validation}

In order to confirm the theoretical predictions, an experiment using the supporting concept proposed was performed. The goal of this experiment is to evaluate the isolation performance of the system in the vertical direction. In fact, so far we only developed and built one vertical inertial sensor. Consequently, no test has been performed on the horizontal direction. A general view of the extended structure for a potential 6 d.o.f. isolation experiment is shown in figure 10.(a). The dimensions of the structure used in this experimental validation and some additional information are listed in table 1. Figure 10.(b) shows one leg of the structure with the vertical and horizontal actuators. The 1 d.o.f. experimental active isolation system is composed of an optical inertial sensor [27] and of two piezoelectric actuators APA100M from Cedrat Technologies, see figure 10.(c). The isolation system is placed at one extremity of the structure while the other extremity is locked. The signals coming from the sensor are measured by the dSpace MicroLabBox with a sampling frequency of $20 \mathrm{kHz}$. The optical inertial sensor consists of a quadrature homodyne Michelson interferometer coupled with a pendulum $[21,27]$. The interferometer provides two quadrature signals whose phases is proportional to the pendulum motion. Consequently, the arctangent of the ratio of these two quadrature signals provides the measurement of the pendulum motion. The sensor has a resolution of $1 \mathrm{pm} / \mathrm{rtHz}$ at $1 \mathrm{~Hz}$.

Based on the motion measured, the control signal is generated and sent to the piezoelectric actuators through a power amplifier $(25 \mathrm{~V} / 1 \mathrm{~V})$. The extended structure, together with the piezos and the sensor are placed on a rigid TMC table. Finally, one Guralp CMG-6T is fixed on the structure in order to calibrate the sensor and another 
Table 1. Dimensions of the extended structure and of some other useful parameters.

\begin{tabular}{ll}
\hline Parameters & Length $(\mathrm{cm})$ \\
\hline $\mathrm{x}$ & 24 \\
$\mathrm{y}$ & 119 \\
$\mathrm{z}$ & 19 \\
$\mathrm{~d}$ & 12 \\
$\mathrm{~h}$ & 0.5 \\
$\alpha$ & $20^{\circ}$ \\
\hline
\end{tabular}

one is placed on the rigid table as a reference.
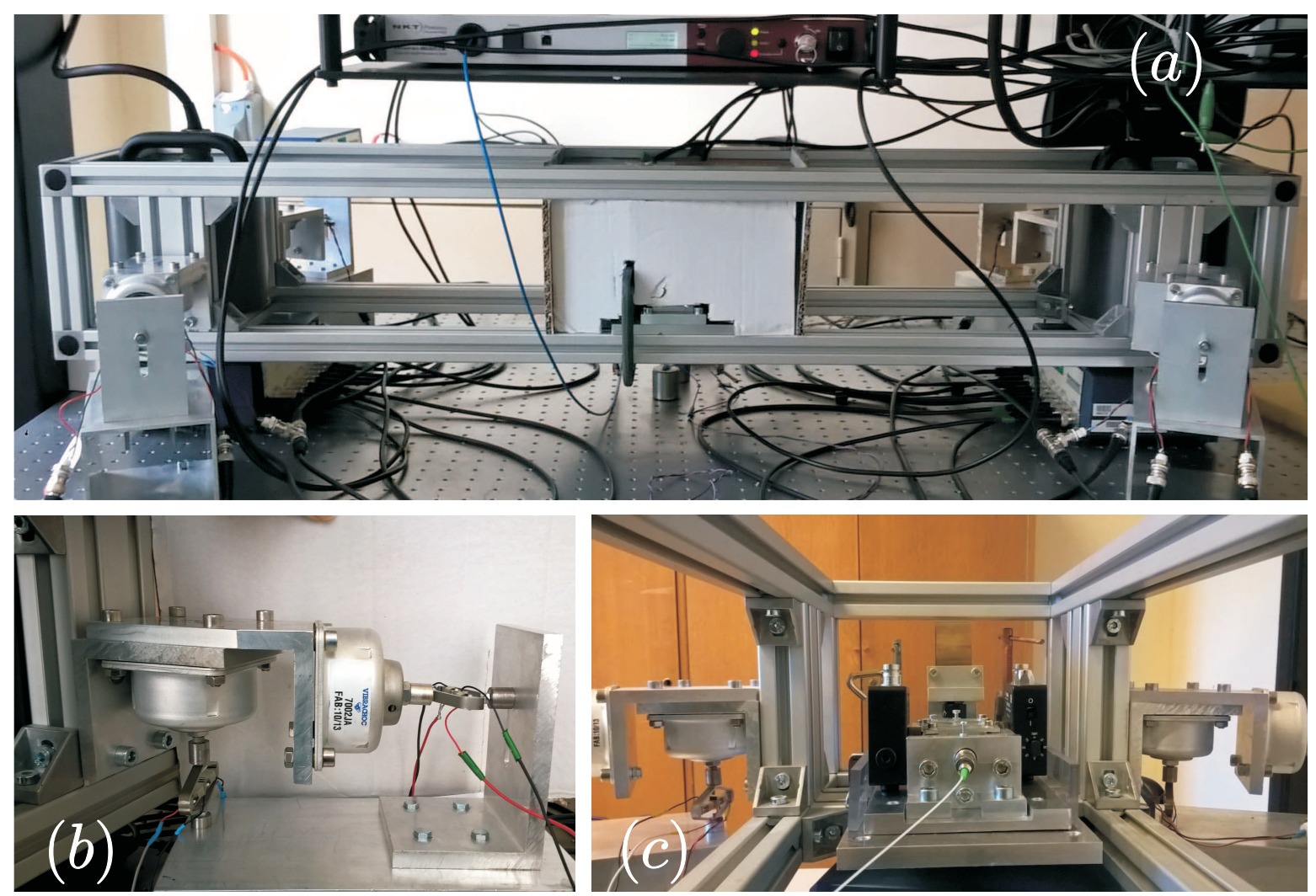

Figure 10. (a) General view of the extended structure for a 6 d.o.f. isolation experiment. On the rigid table: on the right and on the left, the legs holding the structure are visible. In the center of the structure, the optical inertial sensor is placed in a box to reduce thermal fluctuations. The laser source is suspended above the structure. The power amplifier is on the ground below the rigid table. (b) View of one pair of legs: the vertical and horizontal actuators are fixed by the mean of metallic suspensions. (c) View of the 1 d.o.f. experiment: the active isolation stage from inside the extended structure. The optical inertial sensor (center) is fixed thanks to clamps on the structure. The two piezoelectric actuators APA100M are on the left and right side of the structure and hold it. 


\subsection{Inertial control}

The transfer function between the sensor and a vertical actuator is measured by injecting some white noise in the vertical actuators. A fit of the measurement is then generated by system identification (see figure 11).

In comparison with the controller discussed in the previous section, two lags are added in order to extend the bandwidth towards lower frequencies. Their zeros corresponds to the complex conjugate poles of the inertial sensor in order to cancel them

$$
S=\frac{s^{2}+2 \xi \omega_{0} s+\omega_{0}^{2}}{s^{2}+2 \xi_{n} \omega_{n} s+\omega_{n}^{2}}
$$

where $\omega_{0}$ and $\xi$ are the natural frequency and damping ratio of the sensor; $\omega_{n}$ and $\xi_{n}$ are the new natural frequency and damping ratio. Here, $\omega_{0}=2 \pi 6.27 \mathrm{rad} / \mathrm{s}$ and $\xi=4$ $\times 10^{-3}, \omega_{n}=2 \pi 1 \mathrm{rad} / \mathrm{s}$ and $\xi_{n}=0.2$;

In order to verify the stability of the closed loop, the open loop transfer function is estimated based on the controller and the fitted transfer function, see figure 12 . The stability margins confirm that the controller will achieve a good robustness.

Additionnally, a high pass filter $H P$ has been added in order to remove the dc component of the optical inertial sensor signal

$$
\mathrm{HP}=\frac{s}{s+2 \pi 0.1}
$$

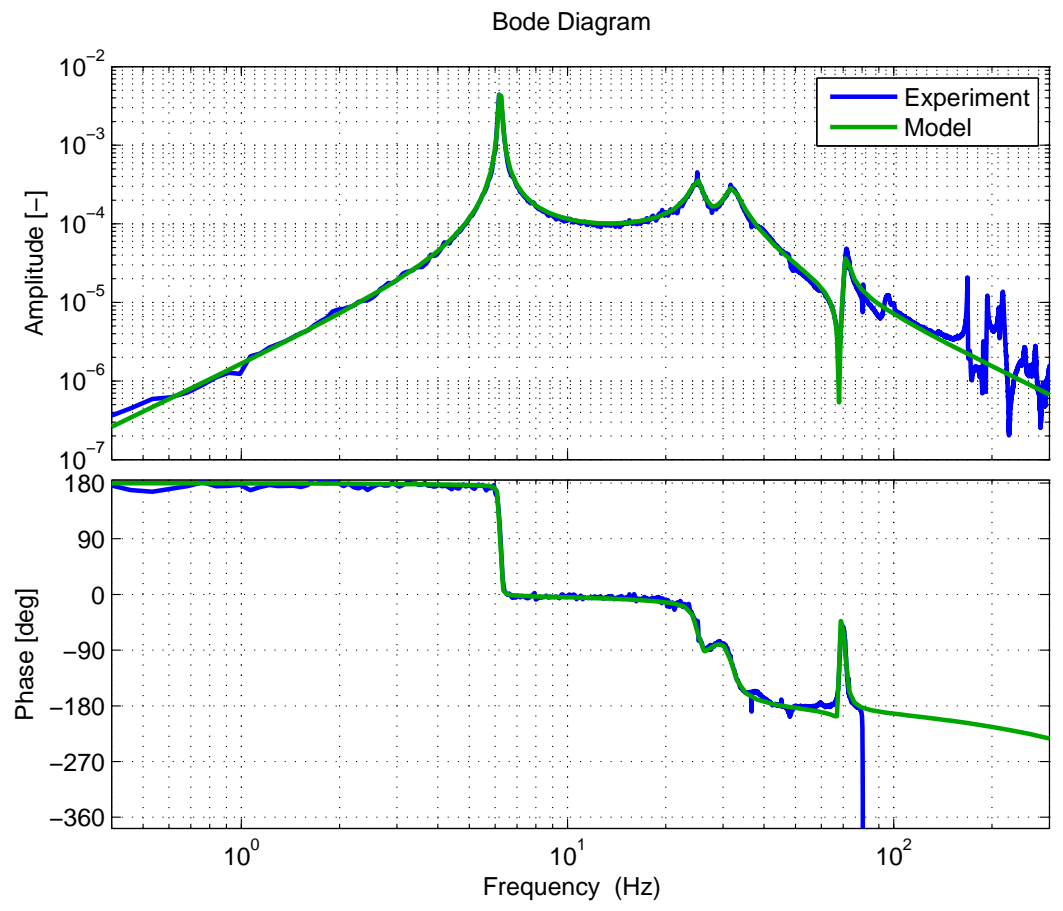

Figure 11. Bode diagram of the experimental transfer function (blue) between the inertial sensor and the actuator and its fit (green). 


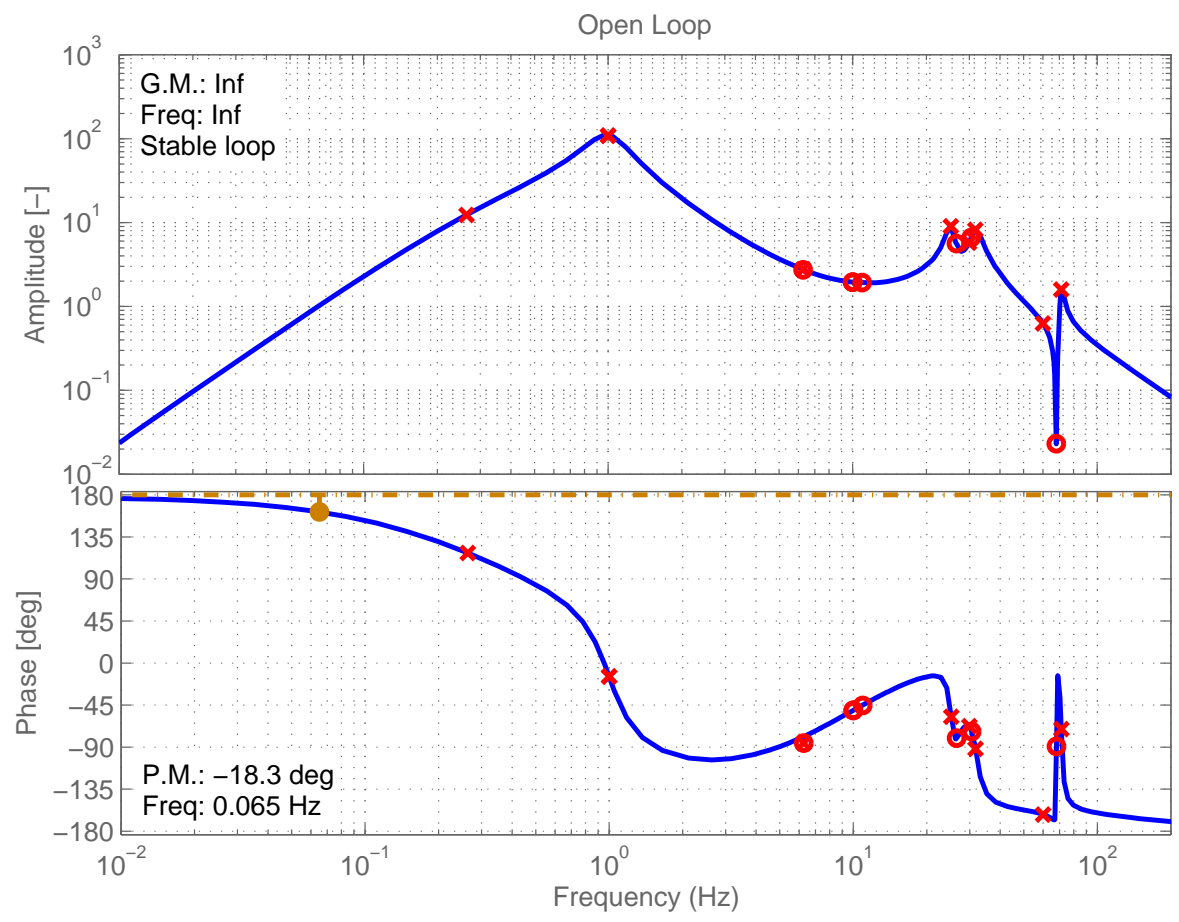

Figure 12. Open loop transfer function between the inertial sensor and the actuator.

\subsection{Noise budgeting}

In this experimental section, the sources of noise must be included in the model as they will limit the performance. The noise sources taken into account come from:

- the sensor resolution $n_{1}$ measured experimentally and including Analog to Digital Conversion (ADC), photodiodes, laser source, etc.;

- the amplification stage $n_{2}$ based on datasheet information and including Digital to Analog Conversion (DAC), power amplification system, etc.;

- the ground motion itself $w$ (measured with the reference Guralp).

In order to see how they combine, a 1 d.o.f. model is developed. Its structure is represented on the block diagram in figure 13. The equation of motion for this 1 d.o.f. model can be expressed as

$$
m \ddot{x}+c_{a} \dot{x}+k_{a} x=f+k_{a} w
$$

where $f$ is the force injected into the actuator and $w$ is the vertical ground motion. By adding the sources of noise in equation (13), the force applied becomes

$$
f=n_{2}-h\left(x+n_{1}\right)
$$

where $h$ is the controller expressed in the time domain. 
In the Laplace domain, the equation of motion becomes

$$
\left(H+m s^{2}+c_{a} s+k_{a}\right) X=k_{a} W+N_{2}-H N_{1}
$$

Which can be expressed as

$$
X=\frac{k_{a}}{H+m s^{2}+c_{a} s+k_{a}} W+\frac{H}{H+m s^{2}+c_{a} s+k_{a}} N_{1}+\frac{1}{H+m s^{2}+c_{a} s+k_{a}} N_{2}
$$

The corresponding Power Spectral Density (PSD) of the residual motion is given by

$\Phi_{x}=\left|\frac{k_{a}}{H-m \omega^{2}+j \omega c_{a}+k_{a}}\right|^{2} \Phi_{w}+\left|\frac{H}{H-m \omega^{2}+j \omega c_{a}+k_{a}}\right|^{2} \Phi_{n_{1}}+\left|\frac{1}{H-m \omega^{2}+j \omega c_{a}+k_{a}}\right|^{2} \Phi_{n_{2}}$

where $\Phi_{n_{1}}, \Phi_{n_{2}}$ and $\Phi_{w}$ are respectively the PSD of the sensor resolution, the amplification stage and the ground motion. $H$ is the controller used. The resolution and ground motion PSD are measured while the amplification stage noise PSD is derived from the datasheet.

The Amplitude Spectral Density (ASD) $\sqrt{\Phi_{x}}$ evaluated with the model is shown in figure 14 with a control gain of $6 \times 10^{4}$. As at the exit of the DAC the signal is multiplied by 10 and the amplification stage increases the signal by a factor 25 , the real gain at the actuators is $1.5 \times 10^{7}$. Around $1 \mathrm{~Hz}$, the isolation performance seems to be limited by ground motion and sensor resolution. Above $2 \mathrm{~Hz}$, the isolation can be improved by increasing the gain until the saturation limit, as the residual motion is bounded by the ground motion. Finally, above $50 \mathrm{~Hz}$, the amplification stage properties limit the performance.

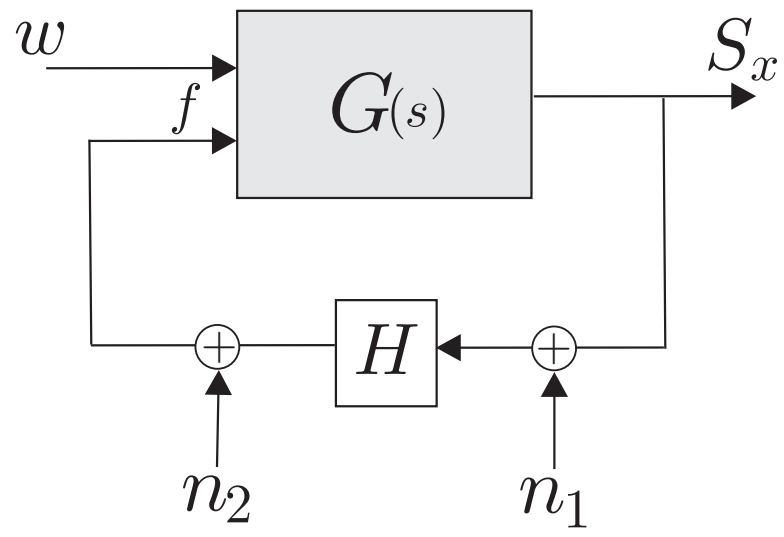

Figure 13. Block diagram of the 1 d.o.f. experiment. The different sources of noise are included: $n_{1}$ corresponds to the resolution of the sensor and $n_{2}$ to the amplification noise. 


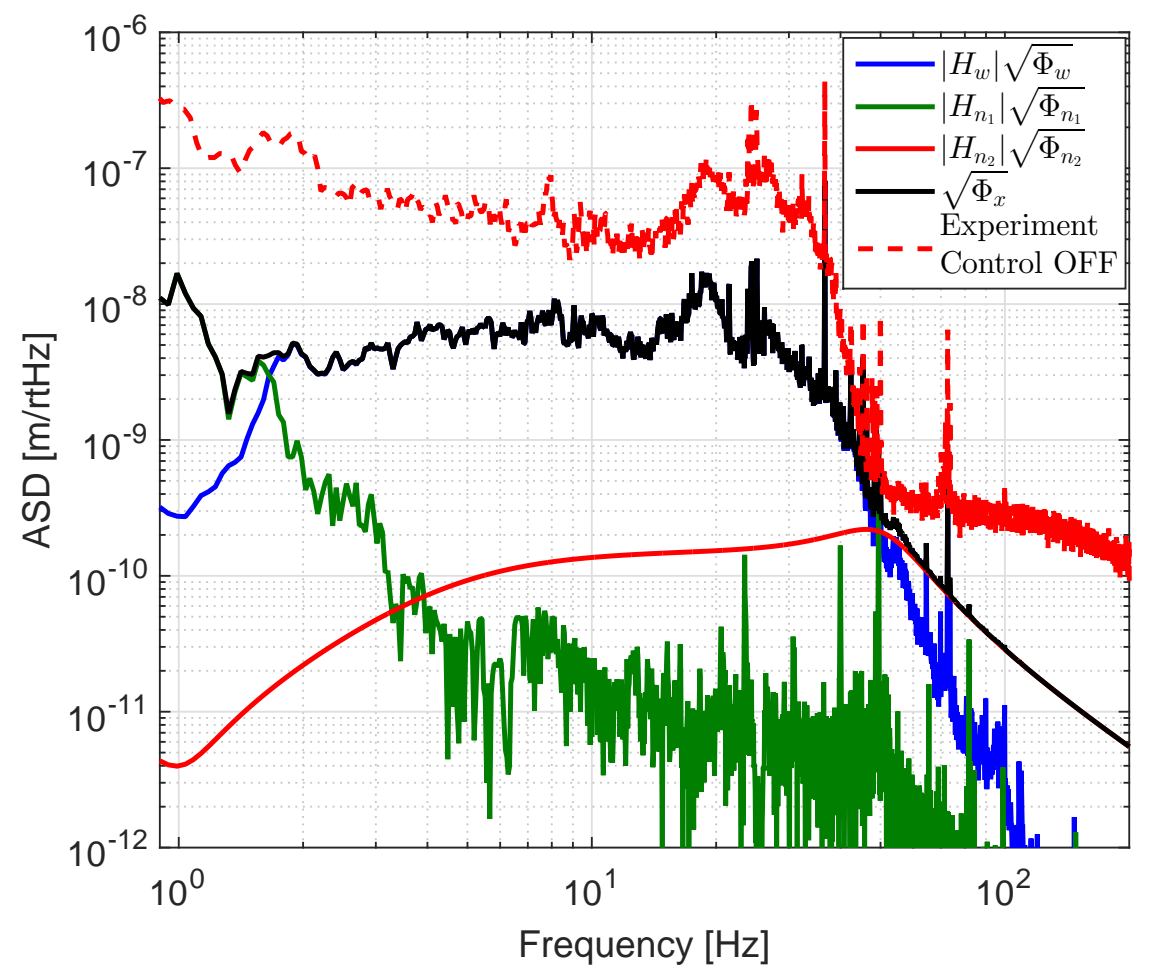

Figure 14. ASD estimation of the contributions of the different sources of noise to the total noise (black): the resolution (green) is dominant around $1 \mathrm{~Hz}$, the ground motion (blue) at low frequency, except around $1 \mathrm{~Hz}$ and the injection noise (red) is dominant above $50 \mathrm{~Hz}$. The ASD of the structure motion measured when no control is applied is also shown (red dashed).

\subsection{Results}

The ASD of the 1 d.o.f. model described above is compared to the experimental ASD measured using the same controller gain in figure 15. As the coherence between the inertial sensor and the Guralp on the table decreases below $1 \mathrm{~Hz}$, the comparison between model and experimental transmissibility is evaluated above $1 \mathrm{~Hz}$; at these frequencies, the model reproduces correctly the signal measured. This is confirmed in figure 16 .

The performance of the controller has been tested during day and night sessions with the controller described in section 5.1 and with a gain of $6 \times 10^{4}$ which corresponds to 1.5 $\times 10^{7}$ at the actuators. The results are presented in Amplitude Spectral Density (ASD) in figure 17. At $1 \mathrm{~Hz}$, an attenuation of a factor 100 is reached. This is in agreement with the performance estimated in the previous sections. On the same graph, the resolution of the inertial sensor is plotted. This illustrates the fact that the isolation is not limited by the resolution of the sensor at any frequency. The experimental performance is summarised in table 2 with the RMS calculated at $1 \mathrm{~Hz}$ resulting from figure 18. 


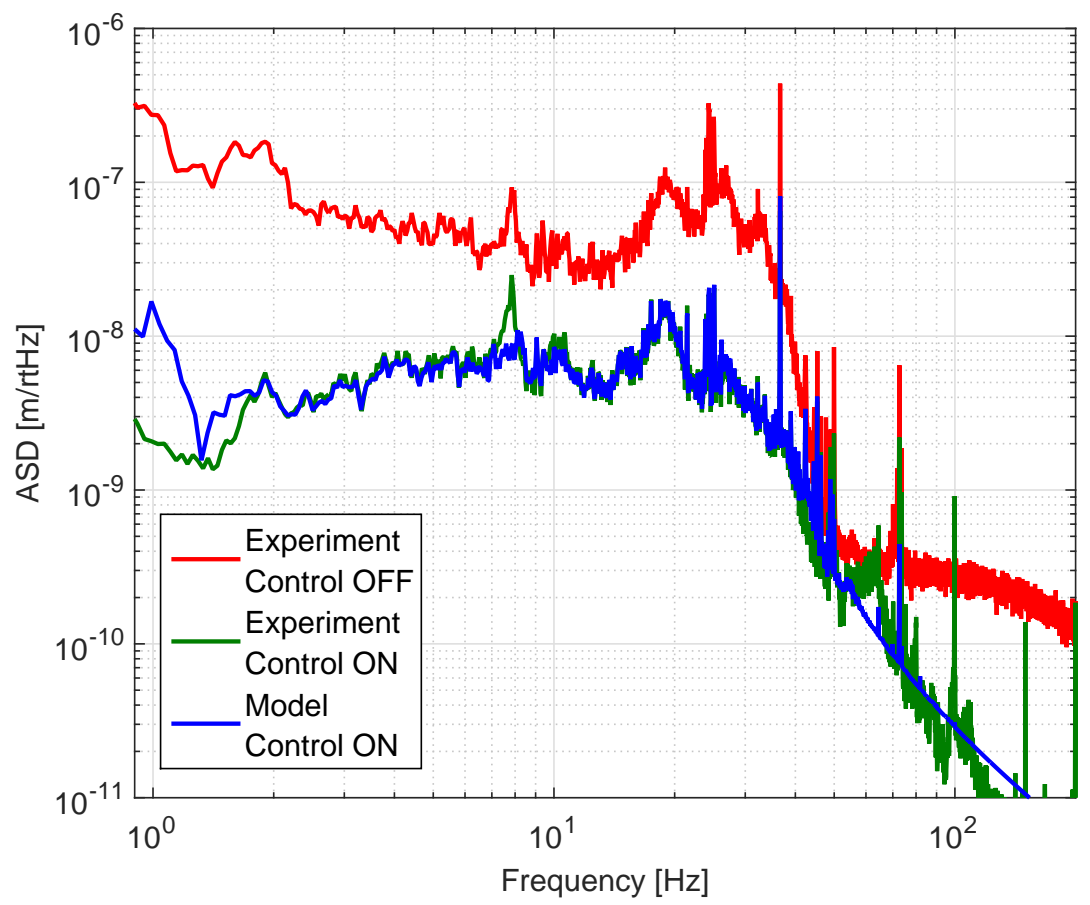

Figure 15. Experimental ASD measured with the inertial sensor on the structure without (red) and with control (green). The blue curve is the estimated ASD obtained with the model using the same controller.

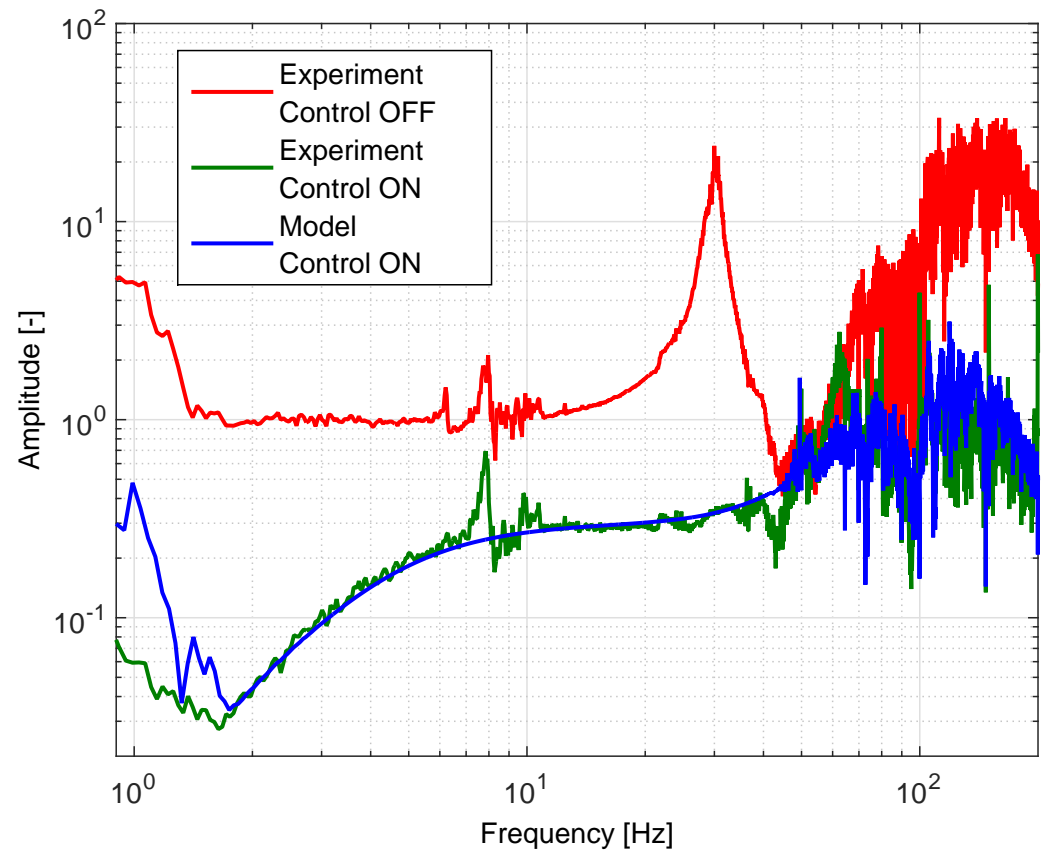

Figure 16. Experimental transfer function between the inertial sensor on the structure and the Guralp on the table without (red) and with control (green). The blue curve is the estimated transfer function obtained with the model using the same controller. 


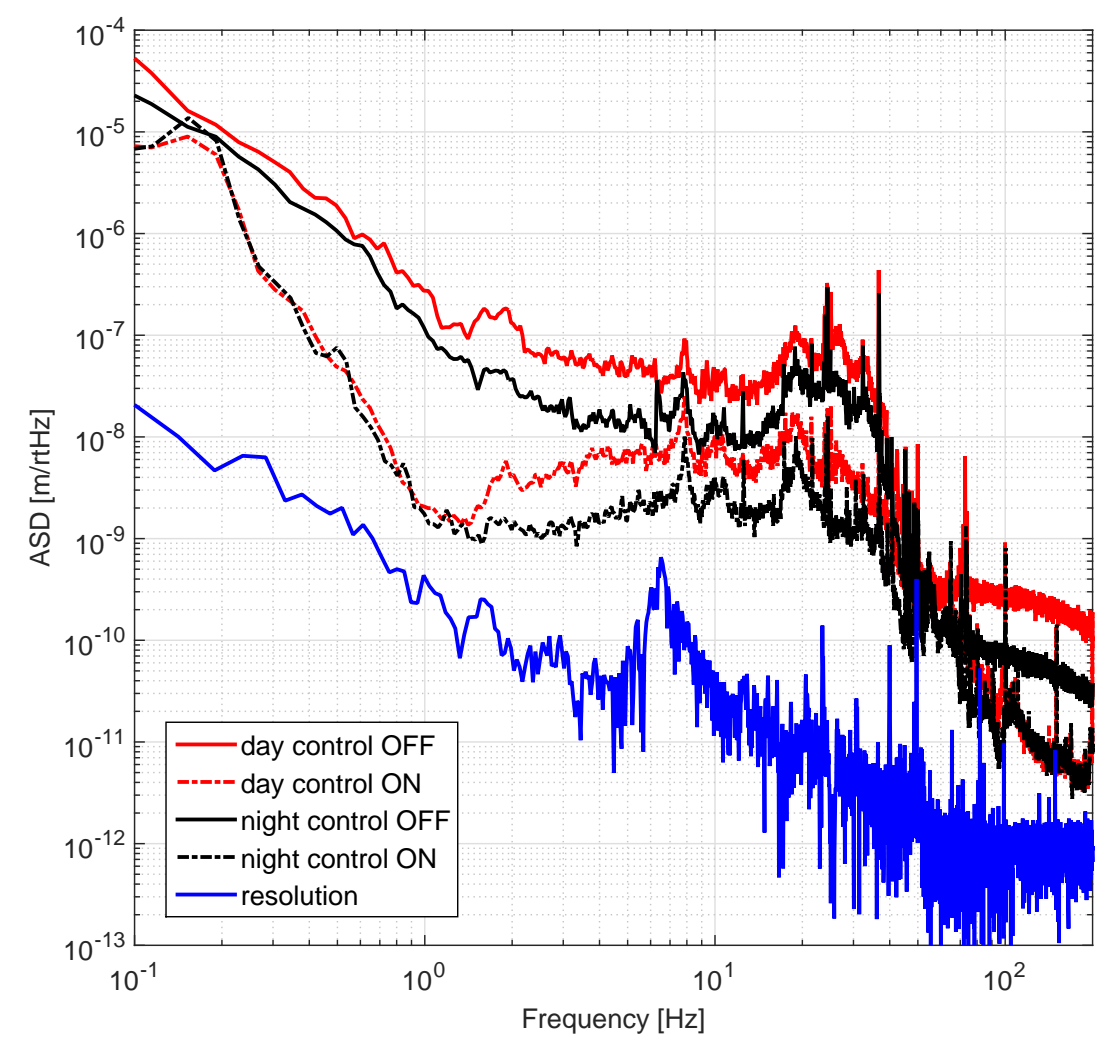

Figure 17. ASD of the day and night experiments using the optical inertial sensor: the day measurements with and without isolation are respectively the dashed red and plain red curves; the night measurements with and without isolation are respectively the dashed black and plain black curves. The blue curve is the resolution of the sensor.

Table 2. RMS of the different experiments calculated at $1 \mathrm{~Hz}$.

\begin{tabular}{lll}
\hline & Day & Night \\
\hline Control OFF & $4.9910^{-7} \mathrm{~m}$ & $2.1410^{-7} \mathrm{~m}$ \\
Control ON & $4.3510^{-8} \mathrm{~m}$ & $1.8110^{-8} \mathrm{~m}$ \\
\hline
\end{tabular}

\section{Conclusion}

The paper has presented an active vibration isolation stage, dedicated to support extended payloads. To the best of the author's knowledge, this is the first active isolation system not containing any coil, magnet or elastomer, which makes it fully compatible with accelerator environments. Using the proposed strategy, it has been demonstrated experimentally that transmitted motion can be reduced by more than two orders of magnitude $(40 \mathrm{~dB})$ when the controller is turned on, in a frequency range extending in the sub-Hz domain. Transposing this high performance in the quiet environment of a particle accelerator, the strategy should allow to stabilize at the sub-nanometer level the electromagnets of the future generation of particle colliders as for instance the CLIC and ILC colliders. 


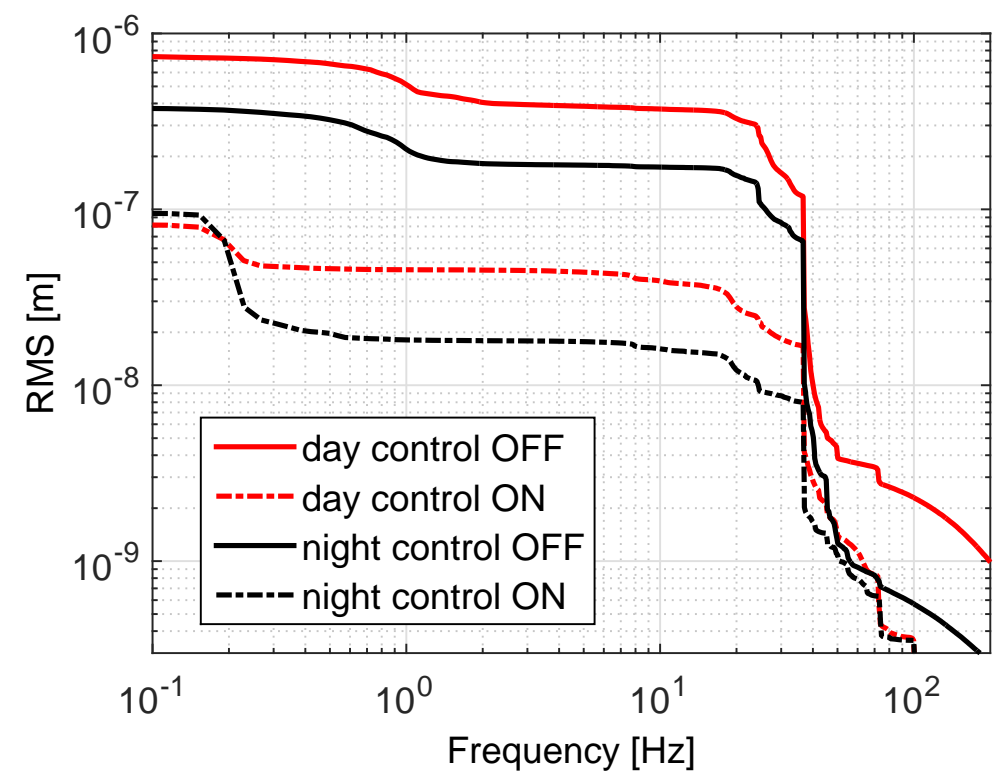

Figure 18. RMS of the day and night experiments: the day measurements with and without isolation are respectively the dashed red and plain red curves; the night measurements with and without isolation are respectively the dashed black and plain black curves.

Although the inertial sensor does not meet sensor noise requirements to potentially improve the gravitational wave detectors active platforms, the prototype lays out technology of interest for a vacuum compatible and magnetic insensitive vertical interferometric sensor, which may be of high interest for the improvement of active isolation stages used in gravitational wave detectors.

\section{Acknowledgments}

The authors gratefully acknowledge the Brussels Capital Region (Innoviris) and the F.R.S.-FNRS for funding this research (grant agreement F.4536.17). 


\section{References}

[1] Butler H 2012 Control Engineering Practice 20453 - 464 ISSN 0967-0661 special Section: \{IFAC $\}$ Symposium on Advanced Control of Chemical Processes - \{ADCHEM\} 2009 URL http://www.sciencedirect.com/science/article/pii/S0967066111002565

[2] Carter A, King G, Ulrich T, Hasley W, Alchenberger D and Perkins T 2007 Applied Optics 46(3) $421-427$

[3] De Angelis M, Bertoldi A, Cacciapuoti L, Giorgini A, Lamporesi G, Prevedelli M, Saccorotti G, Sorrentino F and Tino G 2008 Measurement Science and Technology 20022001

[4] Matichard F e a 2015 Classical and Quantum Gravity 32185003

[5] Bertolini A 2007 Measurement Science and Technology 182293

[6] Montag C 1996 Nuclear Instruments and Methods in Physics Research A 378 396-375

[7] Collette C, Artoos K, Kuzmin A, Janssens S, Sylte M, Guinchard M and Hauviller C 2010 Nuclear Instruments and Methods in Physics Research Section A: Accelerators, Spectrometers, Detectors and Associated Equipment 621 71-78

[8] Collette C, Janssens S, Artoos K, Kuzmin A, Fernandez-Carmona P, Guinchard M, Leuxe R and Hauviller C 2011 Nuclear Instruments and Methods in Physics Research Section A: Accelerators, Spectrometers, Detectors and Associated Equipment

[9] Allison S, Eriksson L, Hendrickson L, Himel T and Seryi A 2001 Active vibration suppression $\mathrm{r}+\mathrm{d}$ for the next linear collider Proceedings of the Particle Accelerator Conference, Chicago

[10] Redaelli S 2003 Stabilization of Nanometre-Size Particle Beams in the Final Focus System of the Compact LInear Collider (CLIC) Ph.D. thesis EPFL, Lausanne

[11] Frisch J, Chang A, Decker V, Doyle E, Eriksson L, Hendrickson L, Himel T, Markiewicz T, Partidge $\mathrm{R}$ and Seryi A 2004 Vibration stabilization of a mechanical model of a x-band linear collider final focus magnet 22nd International Linear Collider Conference, 16-18 August (Germany)

[12] Bolzon B 2007 Etude des vibrations et de la stabilisation l'échelle sous-nanométreique des doublets finaux d'un collisionneur linéaire Ph.D. thesis University of Savoie

[13] Breton R L, Deleglise G, Allibe J, Badel A, Balik G, Caron B, Jeremie A, Lottin J and Vilalte S 2013 Sensors and Actuators A: Physical 20497 - 106 ISSN 0924-4247 URL http://www.sciencedirect.com/science/article/pii/S092442471300469X

[14] Collette C, Tshilumba D, Fueyo-Roza L and Romanescu I 2013 Review of Scientific Instruments 84023302

[15] Caron B, Balik G, Brunetti L and Jeremie A 2012 Control Engineering Practice 20236 - 247 ISSN 0967-0661 URL http://www.sciencedirect.com/science/article/pii/S0967066111002334

[16] Collette C, Janssens S and Tshilumba D 2012 Nuclear Instruments and Methods in Physics Research Section A: Accelerators, Spectrometers, Detectors and Associated Equipment

[17] Danisi A, Losito R and Masi A 2015 Measurement Science and Technology 26094002

[18] Mukherjee B, Rybka D, Makowski D, Lipka T and Simrock S 2007 Measurement Science and Technology 182387

[19] Inaudi D, Glisic B, Fakra S, Billan J, Redaelli S, Perez J G and Scandale W 2001 Measurement Science and Technology $\mathbf{1 2} 887$

[20] Caponero M, Colonna D, Montanini R and Pirrotta S 2006 Measurement Science and Technology 171601

[21] Collette C, Nassif F, Amar J, Depouhon C and Gorza S P 2015 Sensors and Actuators A: Physical $22472-77$

[22] Matichard F e a 2015 Precision Engineering 40 273-286

[23] Matichard F e a 2015 Precision Engineering 40 287-297

[24] Schubert D, Beard A and von Flotow A 1994 SPIE 2264

[25] Vervoordeldonk M and Stoutjesdijk H 2006 Recent developments, a novel active isolation concept (6th euspen International Conference, Baden bei Wien)

[26] Stewart D 1965 Proceedings of the institution of mechanical engineers 180 371-386 
[27] Watchi J, Ding B, Matichard F and Collette C 2016 Development of a high-resolution optical inertial sensor for sub-hz seismic isolation ((ISMA2016 International Conference on Noise and Vibration Engineering, 19-21 September 2016 (Leuven, Belgium))) 